\title{
Vitamin D intake and serum vitamin D in ethnically diverse urban schoolchildren
}

\author{
Lauren E Au ${ }^{1, *}$, Christina D Economos ${ }^{1}$, Elizabeth Goodman ${ }^{2}$, Aviva Must ${ }^{3}$,
} Virginia R Chomitz ${ }^{1}$ and Jennifer M Sacheck ${ }^{1}$

${ }^{1}$ Friedman School of Nutrition Science and Policy, Tufts University, 150 Harrison Avenue, Boston, MA 02111 , USA: ${ }^{2}$ Center for Child and Adolescent Health Policy, Massachusetts General Hospital for Children, Boston, MA, USA: ${ }^{3}$ Department of Public Health \& Community Medicine, Tufts University School of Medicine, Boston, MA, USA

Submitted 18 October 2011: Final revision received 13 February 2012: Accepted 30 March 2012: First published online 3 August 2012

\begin{abstract}
Objective: Low serum vitamin D, which largely affects ethnic minorities, is associated with obesity and other chronic diseases. Little is known about racial/ ethnic differences in intake, particularly in children, or if any differences are associated with differences in serum 25-hydroxyvitamin D (25(OH)D). The objective of the present study was to determine whether racial/ethnic differences in dietary vitamin D intake exist and whether they explain differences in 25(OH)D.

Design: Vitamin D intakes (Block Kids 2004 FFQ) and 25(OH)D were measured. Race/ethnicity was parent-reported (white (37.9\%), Hispanic (32.4\%), black (8.3\%), Asian (10.3\%), multi-racial/other (11.0\%)). Multivariable analyses were conducted to examine the associations among dietary vitamin $\mathrm{D}$ and race/ethnicity, as well as $25(\mathrm{OH}) \mathrm{D}$, independent of BMI $Z$-score and other covariates.

Setting: Elementary/middle schools in Somerville, MA, USA, during January-April 2010.

Subjects: Schoolchildren ( $n$ 145) in 4th-8th grade.

Results: Only $2 \cdot 1 \%$ met the 2011 RDA $(15 \mu \mathrm{g} / \mathrm{d}(600 \mathrm{IU} / \mathrm{d}))$. Average dietary intake was 3.5 (sD 2.2) $\mu \mathrm{g} / \mathrm{d}(140$ (sD 89.0) IU/d). No racial/ethnic differences in intake were evident. Most (83.4\%) were 25(OH)D deficient $(<20 \mathrm{ng} / \mathrm{ml} ; 16 \cdot 0(\mathrm{sD} 6 \cdot 5) \mathrm{ng} / \mathrm{ml})$. In ANOVA post boc analyses, 25(OH)D levels were lower in Hispanics than whites $(14 \cdot 6$ (sD $6 \cdot 1) \mathrm{ng} / \mathrm{ml} v \cdot 17.9(\mathrm{sD} 4 \cdot 6) \mathrm{ng} / \mathrm{ml} ; P<0 \cdot 01)$. Dietary vitamin D was associated with $25(\mathrm{OH}) \mathrm{D}$ overall $(P<0 \cdot 05)$, but did not explain the racial/ethnic differences in 25(OH)D.

Conclusions: Most children in this north-east US sample did not meet dietary recommendations for vitamin D and were vitamin D deficient. Dietary vitamin D did not explain the difference in $25(\mathrm{OH}) \mathrm{D}$ between Hispanic and white children. Further research is needed to determine if changes in dietary vitamin D by race/ethnicity can impact 25(OH)D levels.
\end{abstract}

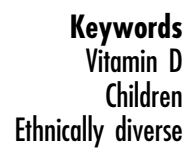

Throughout life, vitamin D is important for Ca absorption and bone growth ${ }^{(1)}$. Emerging evidence suggests that low vitamin D levels are also associated with obesity, diabetes and CVD, and that ethnic minorities are most affected ${ }^{(2)}$. Recently, vitamin D has received growing attention due to the increased awareness of possible deficiencies among certain racial/ethnic populations at risk of vitamin $\mathrm{D}$ deficiency $^{(3,4)}$.

Various studies have found that attaining optimal serum 25-hydroxyvitamin D (25(OH)D) concentrations is more challenging for certain racial/ethnic groups ${ }^{(2)}$. Ethnic minorities have significantly higher rates of vitamin D deficiency $(25(\mathrm{OH}) \mathrm{D}<20 \mathrm{ng} / \mathrm{ml})$ than their white counterparts (44-49\% $v .10 \%$, respectively, in children) ${ }^{(2)}$. Twenty per cent of children aged 6-11 years in recent cycles of the US National Health and Nutrition Examination Survey (NHANES), 2003-2004 and 2005-2006, were reported to have serum $25(\mathrm{OH}) \mathrm{D}$ concentrations $<20 \mathrm{ng} / \mathrm{ml}^{(5)}$, with African-American children having higher rates of vitamin $\mathrm{D}$ deficiency than Caucasian children $(51 \% \quad v$. 9\%). Furthermore, a cross-sectional clinic-based study of 307 children in Boston found that $52 \%$ of African-American and Hispanic children had deficient serum $25(\mathrm{OH}) \mathrm{D}$ levels ${ }^{(6)}$.

Because of the growing recognition of populations at risk of vitamin $\mathrm{D}$ deficiency and the health benefits associated with vitamin D, the Institute of Medicine (IOM) recently revised its recommendations for Dietary Reference Intake (DRI) for vitamin D. The IOM recommended daily vitamin D intakes to be increased from $5 \mu \mathrm{g}$ (200 IU; 
the previous Adequate Intake (AI)) to $10 \mu \mathrm{g}$ (400 IU) to meet the needs of half of children aged 1-18 years and to $15 \mu \mathrm{g}$ (600 IU) daily to meet the needs of $97 \cdot 5 \%$ of these children ${ }^{(7)}$. These new values (10 and $15 \mu \mathrm{g}$ daily) define the Estimated Average Requirement (EAR) and the RDA, respectively ${ }^{(8)}$. However, few children actually meet these recommendations with diet alone, and intake varies by racial/ethnic group. In 1999-2000 NHANES, MexicanAmerican children (69\%) were most likely to meet or exceed the AI, whereas only $48 \%$ of non-Hispanic black children were estimated to meet or exceed the AI levels for vitamin D from food $^{(9)}$. Among children 1-8 years old from the same population, AI levels for vitamin D from food and supplements varied by race/ethnicity with $82 \%$ of MexicanAmerican, $78 \%$ of non-Hispanic white and $66 \%$ of nonHispanic black children meeting or exceeding the AI. Since the new IOM recommendations were released in 2011, racial/ethnic differences in dietary vitamin $\mathrm{D}$ have not been examined against the new dietary recommendations.

Few studies have examined dietary vitamin D and serum vitamin $\mathrm{D}$ in a racially and ethnically diverse population of schoolchildren in a northern latitude of the USA ${ }^{(10)}$. Thus, we chose to examine (i) whether there were racial/ethnic differences in serum vitamin $D$ in a sample of urban schoolchildren and (ii) whether dietary differences in vitamin D were associated with differences in $25(\mathrm{OH}) \mathrm{D}$.

\section{Materials and methods}

\section{Participants}

A cross-sectional design was used to assess the dietary intake of 145 students enrolled in the 2009-2010 Fitness and Metabolic Health (FIT) Study. The FIT Study enrolled children from eight public schools (kindergarten to grade 8) in Somerville, MA, USA, between January and April 2010. Somerville is an ethnically diverse community within the greater Boston metropolitan area, where $68 \%$ of the students in the study receive free (54\%) or reduced-price (14\%) school lunch $^{(11)}$. Schoolchildren were recruited for the study through presentations, flyers and announcements in the schools and received a gift card to a local retailer for their participation. Informed written consent was obtained from parents and children. The protocol was reviewed and approved by the Tufts University Institutional Review Board for inclusion of human subjects. The original sample included 162 children. Underweight children ( $n$ 6), those who reported implausible energy intake $(<2092 \mathrm{~kJ} / \mathrm{d}(<500 \mathrm{kcal} / \mathrm{d})$ or $\left.>20920 \mathrm{~kJ} / \mathrm{d}(>5000 \mathrm{kcal} / \mathrm{d})^{(12)} ; n 8\right)$ and those who did not have accurate serum vitamin $\mathrm{D}$ results $(n 3)$ were excluded from these analyses, leaving a sample of 145 participants for the present study.

\section{Antbropometrics and pubertal status}

Height and weight were measured in triplicate with children in light clothing and without shoes. Height was measured using a portable stadiometer (model 214; Seca Weighing and Measuring Systems, Hanover, MD, USA) with the head in the Frankfort plane made with a right angle height procedure ${ }^{(13)}$ and recorded to the closest $\sim 3 \mathrm{~mm}(1 / 8 \mathrm{in})$. Weight was measured on a portable balance beam scale (Healthometer, Boca Raton, FL, USA) and recorded to the closest $\sim 100 \mathrm{~g}(0 \cdot 25 \mathrm{lb})$. BMI was calculated and then expressed as a Z-score (BMIZ) using the US Centers for Disease Control and Prevention sexspecific growth charts ${ }^{(14)}$. BMIZ was used to represent weight status for analyses.

Pubertal status was assessed by asking the female participants if they had reached menarche (yes/no) and male participants if their voice had changed (yes/no) ${ }^{(15)}$. Answering yes was considered a marker for late puberty.

\section{Race/etbnicity and socio-economic status}

Child race/ethnicity was defined by parental report based on the categories of the Centers for Disease Control and Prevention: white/Caucasian, Mexican/Mexican-American, other Hispanic/Latino, black/African-American, Asian/AsianAmerican/Asian-Indian, Native American/American Indian and multi-racial/multi-ethnic/other ${ }^{(16)}$; and consolidated into five groups: white, Hispanic, black, Asian, multi-racial/other. Participant eligibility for free or reduced-price lunch ( $<185 \%$ of federal household income level) under the National School Lunch Program was provided by the Somerville Public School District and coded as a binary variable for use as an indicator of socio-economic status (SES).

\section{Dietary intake assessment}

Nutritional intakes were assessed using the Block Kids 2004 FFQ (NutritionQuest, Berkeley, CA, USA). The eightpage FFQ asked about frequency and quantity of seventyeight foods eaten, as well as multivitamin intake, in the past week and took approximately $20-30 \mathrm{~min}$ to complete. It has been validated for 8-17-year-old children ${ }^{(17)}$, as well as specifically validated for estimating beverage, $\mathrm{Ca}$ and vitamin D intakes in children when compared with $3 \mathrm{~d}$ food diaries ${ }^{(18)}$. The FFQ was pilot-tested for feasibility in a focus group with children in the community of interest before the study took place. A registered dietitian and a trained graduate student administered the FFQ during the school semester between January and April 2010 within one to two weeks of the blood draw. Children were also provided with a separate portion size picture attachment to improve portion size estimation. The collected dietary data were quantified by NutritionQuest as the daily intake in grams (or millilitres for liquids) and further summarized into daily intakes of energy and nutrients using an algorithm from NutritionQuest. Additional vitamin D contribution from foods and beverages was requested separately from NutritionQuest. In addition to the FFQ, to assess vitamin D intake from supplements, parents reported their child's use and frequency of supplement intake. If a parent reported that 
their child took a multivitamin or vitamin D-containing supplement, the use and frequency were compared with the child's response on the FFQ. If the parent's response differed from the child's response on the FFQ, the parent response was taken. Vitamin D intake was assessed according to the $1997 \mathrm{IOM}^{(19)}$ AI recommendations and the $2011 \mathrm{IOM}^{(7)}$ EAR and RDA recommendations; $5 \mu \mathrm{g} / \mathrm{d}$ (200 IU/d), $10 \mu \mathrm{g} / \mathrm{d}(400 \mathrm{IU} / \mathrm{d})$ and $15 \mu \mathrm{g} / \mathrm{d}(600 \mathrm{IU} / \mathrm{d})$, respectively.

\section{5-Hydroxyvitamin D}

Phlebotomy was conducted at school between 07.00 and 08.00 hours, after a $12 \mathrm{~h}$ overnight fast, during late winter (January to March 2010). Participants were asked if they had consumed any beverages or foods before the morning blood draw. Blood was drawn, in private, by a trained phlebotomist from the antecubital vein. All samples were centrifuged, aliquotted and stored at $-80^{\circ} \mathrm{C}$ until analysis. Total $25(\mathrm{OH}) \mathrm{D}$ was determined by a competitive binding RIA (DiaSorin Inc., Stillwater, MN, USA). The intra- and inter-assay CV are 8.6-11.7\% and 8.2-11.0\%, respectively. Vitamin D deficiency status was categorized according to the $2011 \mathrm{IOM}^{(7)}$ report as $25(\mathrm{OH}) \mathrm{D}<20 \mathrm{ng} / \mathrm{ml}$.

\section{Statistical analysis}

All statistical analyses were performed with the statistical software package SPSS $17 \cdot 0$ for Windows (SPSS Inc.). To address the influence of outlying values, nutrient data were winsorized to the 1st and 99th percentiles and did not alter results of any statistical significance testing.

To determine ethnic differences in relevant anthropometrics, demographics, serum $25(\mathrm{OH}) \mathrm{D}$, total energy, dietary vitamin $\mathrm{D}$ and dietary Ca, ANOVA with post hoc analyses was conducted. Exploratory data analysis revealed that serum $25(\mathrm{OH}) \mathrm{D}$ was right skewed, thus the value for $25(\mathrm{OH}) \mathrm{D}$ was transformed by taking the natural logarithm for statistical analyses.

Linear regression analyses were performed to determine the association between race/ethnicity and dietary vitamin D. First, in unadjusted analyses, the regression of dietary vitamin $\mathrm{D} v$. each covariate was performed separately. Multiple linear regression analysis was then performed to evaluate the adjusted associations between race/ethnicity and dietary vitamin $\mathrm{D}$ adjusting for age, gender, BMIZ and SES.

To examine the association between dietary vitamin $\mathrm{D}$ and serum 25(OH)D, Spearman's correlation tests and multiple linear regression analysis were performed. The initial multivariable model included all explanatory variables (dietary vitamin D from foods, vitamin D from supplements, BMIZ, age, gender, pubertal status, race/ ethnicity and SES). Interaction terms between race/ethnicity and dietary vitamin $\mathrm{D}$ were then examined. In order to refine the model, non-significant interaction terms $(P>0 \cdot 05)$ were removed.

Owing to the small number of participants ( $n$ 24, $16.6 \%)$ who had serum $25(\mathrm{OH}) \mathrm{D}$ above the recommended level ( $\geq 20 \mathrm{ng} / \mathrm{ml}$ ), logistic regression was unable to be performed for this analysis. A $P$ value of 0.05 was used to determine statistical significance throughout.

\section{Results}

Participants' characteristics are presented in Table 1, both for the total sample ( $n$ 145) and stratified by race/ethnic group. Children's ages ranged from 9 to 15 years $(60 \cdot 7 \%$ female). Over $60 \%$ of the participants were from racial/ ethnic minorities $(32 \cdot 4 \%$ Hispanic, $8 \cdot 3 \%$ black, $10 \cdot 3 \%$ Asian and $11.0 \%$ multi-racial). Most of the Hispanic children were from El Salvador, Puerto Rico and Guatemala. Weight, BMIZ and SES differed between groups $(P<0.05, P<0.05$ and $P<0.001$, respectively). Over half of the children in the study were overweight or obese ( $51 \cdot 0 \%$ : $70 \cdot 2 \%$ of Hispanics, $50 \cdot 0 \%$ of multi-racial children, $43 \cdot 6 \%$ of whites, $33.3 \%$ of blacks and $33.3 \%$ of Asians $(P<0 \cdot 05)$. In comparison with white children, Hispanic children had higher BMIZ $(P<0 \cdot 05)$; however, BMIZ did not differ significantly between other groups.

Table 1 Demographic and anthropometric characteristics by ethnicity among participants in the 2010 Fitness and Metabolic Health (FIT) Study who completed a Block Kids 2004 FFQ ( $n$ 145)

\begin{tabular}{|c|c|c|c|c|c|c|c|c|c|c|c|c|c|}
\hline & \multicolumn{2}{|c|}{$\begin{array}{l}\text { Total } \\
(n \text { 145) }\end{array}$} & \multicolumn{2}{|c|}{$\begin{array}{l}\text { White } \\
(n 55)\end{array}$} & \multicolumn{2}{|c|}{$\begin{array}{c}\text { Hispanic } \\
\text { (n 47) }\end{array}$} & \multicolumn{2}{|c|}{$\begin{array}{l}\text { Black } \\
(n 12)\end{array}$} & \multicolumn{2}{|c|}{$\begin{array}{l}\text { Asian } \\
(n 15)\end{array}$} & \multicolumn{2}{|c|}{$\begin{array}{l}\text { Multi-racial/other } \\
\quad(n 16)\end{array}$} & \multirow[b]{2}{*}{$P$} \\
\hline & Mean & SD & Mean & SD & Mean & SD & Mean & SD & Mean & SD & Mean & SD & \\
\hline Age (years)* & $11 \cdot 4$ & $1 \cdot 6$ & $10 \cdot 9$ & 1.5 & $11 \cdot 7$ & 1.5 & $11 \cdot 3$ & $1 \cdot 8$ & $11 \cdot 3$ & $1 \cdot 7$ & $12 \cdot 1$ & $1 \cdot 7$ & 0.06 \\
\hline Height $(m)^{\star}$ & $1 \cdot 51$ & $0 \cdot 10$ & $1 \cdot 50$ & 0.09 & $1 \cdot 52$ & $0 \cdot 11$ & 1.55 & $0 \cdot 10$ & $1 \cdot 46$ & 0.09 & 1.55 & 0.09 & $0 \cdot 10$ \\
\hline Weight $(\mathrm{kg})^{*}$ & $51 \cdot 2$ & $16 \cdot 2$ & $47 \cdot 3$ & $15 \cdot 6$ & $56 \cdot 0$ & $16 \cdot 7$ & $49 \cdot 8$ & $13 \cdot 9$ & $46 \cdot 4$ & $15 \cdot 1$ & $56 \cdot 3$ & $15 \cdot 6$ & 0.03 \\
\hline BMI Z-score* & $0 \cdot 86$ & $1 \cdot 0$ & 0.59 & $1 \cdot 0$ & $1 \cdot 30 \ddagger$ & 0.9 & 0.51 & $1 \cdot 2$ & 0.68 & $1 \cdot 1$ & $1 \cdot 00$ & 0.9 & 0.01 \\
\hline Female (\%)† & \multicolumn{2}{|c|}{$60 \cdot 7$} & \multicolumn{2}{|c|}{$60 \cdot 0$} & \multicolumn{2}{|c|}{$57 \cdot 5$} & \multicolumn{2}{|c|}{$50 \cdot 0$} & \multicolumn{2}{|c|}{$60 \cdot 0$} & \multicolumn{2}{|c|}{$81 \cdot 3$} & 0.46 \\
\hline Pubertal (\% yes)† ( $n$ 144) & \multicolumn{2}{|c|}{$48 \cdot 6$} & \multicolumn{2}{|c|}{$37 \cdot 0$} & \multicolumn{2}{|c|}{$53 \cdot 2$} & \multicolumn{2}{|c|}{$66 \cdot 7$} & \multicolumn{2}{|c|}{$40 \cdot 0$} & \multicolumn{2}{|c|}{$68 \cdot 8$} & $0 \cdot 10$ \\
\hline $\begin{array}{l}\text { Free/reduced-price school } \\
\text { lunch }(\%) \dagger\end{array}$ & \multicolumn{2}{|c|}{$73 \cdot 8$} & \multicolumn{2}{|c|}{$41 \cdot 2$} & \multicolumn{2}{|c|}{100} & \multicolumn{2}{|c|}{$91 \cdot 7$} & \multicolumn{2}{|c|}{$66 \cdot 7$} & \multicolumn{2}{|c|}{100} & $<0.001$ \\
\hline
\end{tabular}

${ }^{*}$ Analysed with one-way ANOVA test.

tAnalysed with the Pearson $\chi^{2}$ test.

¥Mean value was significantly different from that of whites $(P<0.01$, ANOVA with post hoc Bonferroni). 
Serum 25(OH)D levels, total energy intake, dietary vitamin $\mathrm{D}$ intake from foods, vitamin $\mathrm{D}$ intake from supplements, total vitamin D intake and dietary Ca intake are summarized for each racial/ethnic group in Table 2 . Hypovitaminosis D was very common $-83.4 \%$ of children were vitamin D deficient $(<20 \mathrm{ng} / \mathrm{ml})$. Post hoc tests suggested that serum 25(OH)D was lower in Hispanics than whites $(14 \cdot 6$ (sD 6.1) $\mathrm{ng} / \mathrm{ml} v .17 \cdot 9$ (sD $4 \cdot 6) \mathrm{ng} / \mathrm{ml}$; $P<0 \cdot 01$ ). Average dietary vitamin $\mathrm{D}$ intake was 3.5 (sD 2.2) $\mu \mathrm{g} / \mathrm{d}(140$ (SD 89.0) IU/d). About one-third (31·7\%) met the 1997 IOM recommendation of $5 \mu \mathrm{g} / \mathrm{d}(200 \mathrm{IU} / \mathrm{d})$. Only $2 \cdot 1 \%$ met the $15 \mu \mathrm{g} / \mathrm{d}(600 \mathrm{IU} / \mathrm{d})$ current RDA recommendation. Of the twenty-five children who reported taking a supplement with vitamin D, only fifteen of them reported taking it every day (10\%). Furthermore, the few children (n3) who met the $15 \mu \mathrm{g} / \mathrm{d}$ recommendation took a vitamin D-containing multivitamin with $10 \mu \mathrm{g} / \mathrm{d}$.

Table 3 shows the results of the regression analyses examining correlates of dietary vitamin D. No racial/ ethnic differences in intake were evident in unadjusted analyses or after adjustment for BMIZ, age, gender and sociodemographic characteristics.

No association was found for vitamin D intake from supplements and serum 25(OH)D in the twenty-five children who took a vitamin D-containing supplement. While dietary vitamin $\mathrm{D}$ intake was associated with serum $25(\mathrm{OH}) \mathrm{D}$ in bivariate analysis $(R=0 \cdot 19, \quad P<0 \cdot 05)$, in multivariable regression dietary vitamin $\mathrm{D}$ was not associated with serum 25(OH)D. However, race/ethnicity was associated with serum vitamin D: Hispanic $(B=-0 \cdot 08$, SE 0.04, $P<0 \cdot 05)$ and black $(B=-0 \cdot 11$, se $0 \cdot 05, P<0 \cdot 05$; data not shown).

\section{Discussion}

Most of the children in the present study were vitamin D deficient; only $16 \%$ of participants had recommended serum 25(OH)D levels. All black children in our study were vitamin D deficient, which is much greater than in previous studies done in similar populations ${ }^{(6,20)}$. Hispanics had lower serum 25(OH)D than whites, which is consistent with findings of earlier studies ${ }^{(21)}$. Asians and blacks also had lower serum 25(OH)D levels than whites; however, due to their low sample sizes, the differences were not significant. These findings suggest that race/ethnicity is a large contributor to serum $25(\mathrm{OH}) \mathrm{D}$, even during late winter when there is limited sunlight exposure and skin colour should, in theory, have less impact on vitamin d status.

The present study found that the average vitamin D intake from foods and supplements was low, with close to $98 \%$ of children failing to meet the new RDA of $15 \mu \mathrm{g} / \mathrm{d}(600 \mathrm{IU} / \mathrm{d})$ and with many children (68.3\%) below the 1997 IOM dietary vitamin D recommendation of $5 \mu \mathrm{g} / \mathrm{d}(200 \mathrm{IU} / \mathrm{d})$, which was the current recommendation at the time of the study. There were no racial/ethnic differences in vitamin D intake in the unadjusted and adjusted analyses. In bivariate

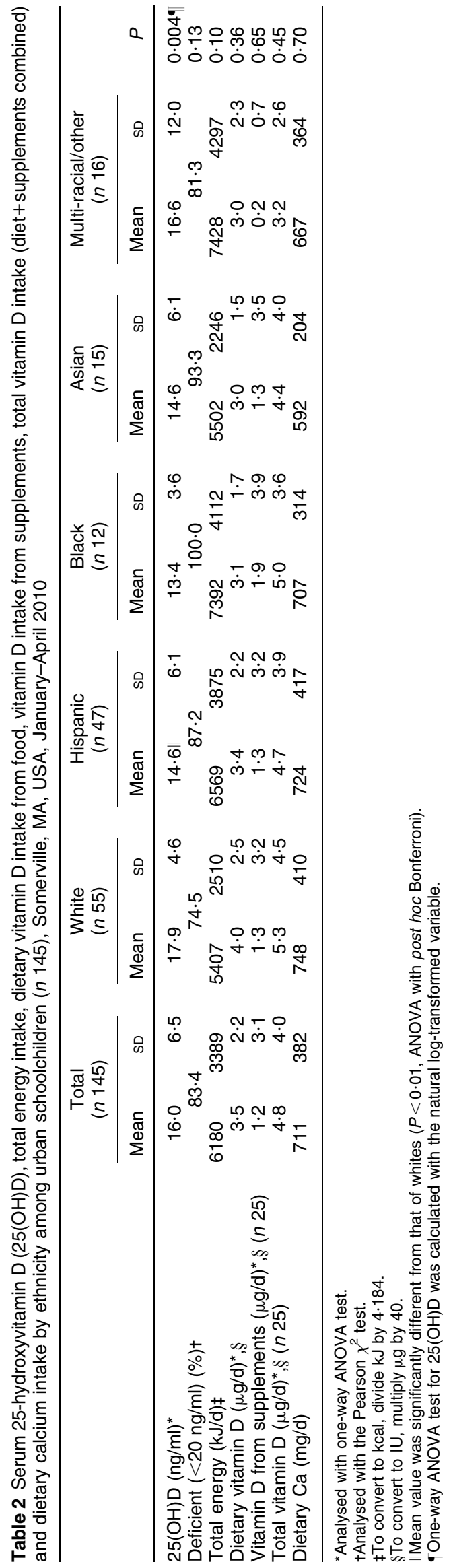


Table 3 Associations* between race/ethnicity and dietary vitamin D intake in urban schoolchildren ( $n$ 145), Somerville, MA, USA, January-April 2010

\begin{tabular}{|c|c|c|c|c|c|c|}
\hline & \multicolumn{3}{|c|}{ Unadjusted } & \multicolumn{3}{|c|}{ Adjusted } \\
\hline & $B$ & $95 \% \mathrm{Cl}$ & $P$ & $B$ & $95 \% \mathrm{Cl}$ & $P$ \\
\hline \multicolumn{7}{|l|}{ Ethnicity } \\
\hline White & $t$ & - & - & $t$ & - & - \\
\hline Hispanic & $-21 \cdot 8$ & $-56 \cdot 6,13 \cdot 1$ & 0.22 & $-10 \cdot 3$ & $-54 \cdot 1,33 \cdot 5$ & 0.64 \\
\hline Black & $-35 \cdot 2$ & $-91 \cdot 2,20 \cdot 8$ & 0.22 & $-32 \cdot 1$ & $-91 \cdot 7,27 \cdot 5$ & 0.29 \\
\hline Asian & $-37 \cdot 9$ & $-89 \cdot 1,13 \cdot 2$ & $0 \cdot 15$ & $-34 \cdot 0$ & $-85 \cdot 9,17 \cdot 9$ & 0.20 \\
\hline Multi-racial/other & $-38 \cdot 4$ & $-88 \cdot 3,11 \cdot 5$ & $0 \cdot 13$ & $-29 \cdot 6$ & $-85 \cdot 4,26 \cdot 2$ & $0 \cdot 30$ \\
\hline Age & $-10 \cdot 6$ & $-19 \cdot 6,-1 \cdot 52$ & 0.02 & $-9 \cdot 36$ & $-18 \cdot 9,0 \cdot 17$ & 0.05 \\
\hline
\end{tabular}

analysis, dietary vitamin $\mathrm{D}$ was associated with serum $25(\mathrm{OH}) \mathrm{D}$ during the winter months in this sample of schoolchildren living in the north-eastern USA. The association between dietary vitamin D and serum 25(OH)D levels was also shown in a recent study of 140 healthy 6-12-year-old African-American and Caucasian children during the winter months ${ }^{(20)}$.

Few foods naturally contain vitamin D precursors ${ }^{(1)}$, making vitamin $\mathrm{D}$ difficult to obtain through diet. The flesh of fatty fish, like salmon and tuna, and cod liver oils, are some of the best vitamin D-rich sources ${ }^{(19)}$, yet these are not frequently consumed by children ${ }^{(7)}$. Currently, fortified foods such as milk, juices, yoghurt, bread and breakfast cereals provide most of the vitamin D in the American diet ${ }^{(19,22)}$. Vitamin D can also be provided through dietary supplements and multivitamins; however, these are not often utilized by children and adolescents $^{(23)}$. Low milk consumption and not taking vitamin D supplements were among the risk factors for low vitamin D status identified in the NHANES 2001-2004 survey of children aged 1-21 years ${ }^{(21)}$. In our study, the largest contributor of dietary vitamin D came from milk or milk products; however, overall intake was low. In addition, supplementation was rare, with only $10 \%$ of children reporting taking one every day, which falls far below national patterns ${ }^{(23)}$. Dietary vitamin D intake did not seem to strongly influence vitamin D status in our study, as hypothesized. This could reflect the overall low vitamin D intake in the sample, regardless of race/ethnicity, as well as the very high rate of vitamin D deficiency.

In addition to low dietary vitamin $\mathrm{D}$, several factors could have contributed to the large percentage of children in our study below serum 25(OH)D recommendations. For example, our sample included a large percentage of racial/ ethnic minorities. While we did not have skin colour data available on this sample, darker-skinned ethnic groups have been shown to have lower $25(\mathrm{OH}) \mathrm{D}$ than lighter-skinned ethnic groups living in the same geographic area ${ }^{(24-26)}$ because they have more skin melanin which acts as a UV filter ${ }^{(27)}$. To control for sunlight exposure and differences in absorption due to skin colour, all children were measured during late winter (January-March) when 25(OH)D is likely to be the lowest, given that the sun does not emit sufficient UVB rays at that time to produce vitamin D in the skin in areas of northern latitude ${ }^{(1)}$. In addition, the low serum vitamin $\mathrm{D}$ levels may have been due to the large percentage of overweight/obese children (51.0\%), which is higher than $31 \cdot 8 \%$ of children and adolescents aged $2-19$ years reported in the most recent cycle of NHANES ${ }^{(28)}$. The high level of overweight and obesity in our sample is of importance because adiposity has been hypothesized to be associated with decreased bioavailability of vitamin $\mathrm{D}$ due to its deposition in body fat ${ }^{(6,29,30)}$.

The present study had some limitations. Because it was cross-sectional, the directionality of the reported associations cannot be established, and the sample size of some of the racial/ethnic groups may have been insufficient to detect weak associations. For example, the differences in serum $25(\mathrm{OH}) \mathrm{D}$ were significant between white and Hispanic groups, but not Asians, who had the same levels as the Hispanic group, or blacks, who had lower levels than Hispanics or Asians. Moreover, because the majority of children had low levels of vitamin D intake, it was also difficult to detect differences between groups. These findings suggest that a larger sample size for these racial/ethnic groups is needed in future studies to fully examine racial/ethnic differences in dietary and serum vitamin D. Lastly, dietary assessment is particularly challenging in children, because of their limited ability to remember their diet and their lack of knowledge of food and food preparation ${ }^{31,32)}$. Furthermore, newly vitamin D-fortified foods, such as vitamin D-fortified orange juice, are not included in the 2004 version of the Block Kids FFQ. However, the study's design was strengthened by the use of a validated ${ }^{(17,18)}$ FFQ for estimating vitamin $\mathrm{D}$ intake in this population and was pilot-tested in the community of interest. A final strength was that our study population comprised a racially and ethnically diverse sample of schoolchildren from an urban location in the greater Boston metropolitan area, who are likely to be at highest risk of developing vitamin D deficiency due to environmental and socio-economic factors. 


\section{Conclusion}

In summary, the majority of children in this sample from the north-east USA did not meet dietary recommendations for vitamin D intake and were vitamin D deficient. Lower dietary vitamin $\mathrm{D}$ intake was associated with lower $25(\mathrm{OH}) \mathrm{D}$ during late winter in urban schoolchildren, although intake did not explain racial/ethnic difference in $25(\mathrm{OH}) \mathrm{D}$. Further research is needed to determine to what extent changes in dietary vitamin $\mathrm{D}$ by racial/ethnic group can impact $25(\mathrm{OH}) \mathrm{D}$.

\section{Acknowledgements}

This study was funded by the American Heart Association (grant \#0835639D) and supported by the Boston Nutrition Obesity Research Center (grant \#P30 DK46200). L.E.A. also received a US Department of Agricultural Doctoral Fellowship in Obesity (grant \#DAG700). No authors had potential conflicts of interest. All authors contributed intellectually to the research aims; L.E.A. and J.M.S. conducted the research; L.E.A analysed the data; L.E.A. wrote the manuscript; and L.E.A. and J.M.S. had primary responsibility for the final content. All authors read and approved the final manuscript. The authors would like to thank the Somerville Public Schools for letting them conduct the research within the schools. In addition, they would like to gratefully acknowledge the support of Kenneth Chui and Peter Bakun at Tufts University for their assistance with data management and analyses; and also thank the Tufts University study staff, especially Erin Sillin, Julius Schneider and the graduate students who helped with data collection.

\section{References}

1. Holick M (2007) Vitamin D deficiency. N Engl J Med 357, 266-281.

2. Renzaho AMN, Halliday JA \& Nowson C (2011) Vitamin D, obesity, and obesity-related chronic disease among ethnic minorities: a systematic review. Nutrition 27, 868-879.

3. Saintonge S, Bang H \& Gerber L (2009) Implications of a new definition of vitamin $\mathrm{D}$ deficiency in a multiracial US adolescent population: the National Health and Nutrition Examination Survey III. Pediatrics 123, 797-803.

4. Weng F, Shults J, Leonard M et al. (2007) Risk factors for low serum 25-hydroxyvitamin D concentrations in otherwise healthy children and adolescents. Am J Clin Nutr 86, $150-158$.

5. Mansbach J, Ginde A \& Carmargo C (2009) Serum 25hydroxyvitamin D levels among US children aged 1 to 11 years: do children need more vitamin D? Pediatrics 124, 1404-1410.

6. Gordon C, DePeter K, Feldman H et al. (2004) Prevalence of vitamin deficiency among healthy adolescents. Arch Pediatr Adolesc Med 158, 531-537.

7. Institute of Medicine (2011) Dietary Reference Intakes for Calcium and Vitamin D. Washington, DC: The National Academies Press.
8. Abrams SA (2011) Vitamin D requirements in adolescents: what is the target? Am J Clin Nutr 93, 483-484.

9. Moore CE, Murphy MM \& Holick MF (2005) Vitamin D intakes by children and adults in the United States differ among ethnic groups. J Nutr 135, 2478-2485.

10. Sacheck J GE, Chui K, Chomitz V et al. (2011) Vitamin D deficiency, adiposity, and cardiometabolic risk in urban schoolchildren. J Pediatr 159, 945-950.

11. Massachusetts Department of Elementary and Secondary Education (2011) School/District Profiles, Somerville (02740000), Selected Populations (2008-09), Students. http://profiles.doe.mass.edu/profiles/student.aspx?orgcode $=$ 02740000\&orgtypecode $=5 \&$ left NavId $=305 \& \&$ fycode $=2009$ (accessed September 2011).

12. Field AE, Peterson KE, Gortmaker SL et al. (1999) Reproducibility and validity of a food frequency questionnaire among fourth to seventh grade inner-city school children: implications of age and day-to-day variation in dietary intake. Public Health Nutr 2, 293-300.

13. Lohman T (1992) Advances in Body Composition Assessment: Current Issues in Exercise Science. Champaign, IL: Human Kinetics.

14. Centers for Disease Control and Prevention (2000) CDC Table for Calculated Body Mass Index Values for Selected Heights and Weights for Ages 2 to 20. http://www.cdc.gov/ nccdphp/dnpa/bmi/00binaries/bmi-tables.pdf (accessed December 2007).

15. Tanner J (1990) Fetus into Man: Physical Growth from Conception to Maturity, 3rd ed. Cambridge, MA: Harvard University Press.

16. Centers for Disease Control and Prevention (2000) Race and Ethnicity Code Set. Washington, DC: CDC; available at http://wonder.cdc.gov/wonder/help/populations/bridgedrace/Directive15.html

17. Cullen K, Watson K \& Zakeri I (2008) Relative reliability and validity of the Block Kids Questionnaire among youth aged 10-17 years. J Am Diet Assoc 108, 862-866.

18. Marshall TA, Eichenberger Gilmore JM, Broffitt B et al. (2008) Relative validity of the Iowa Fluoride Study targeted Nutrient Semi-Quantitative Questionnaire and the Block Kids' Food Questionnaire for estimating beverage, calcium, and vitamin D intakes by children. J Am Diet Assoc 108, 465-472.

19. Standing Committee on the Scientific Evaluation of Dietary Reference Intakes, Food and Nutrition Board, Institute of Medicine (1997) Dietary Reference Intakes for Calcium, Phosphorus, Magnesium, Vitamin D, and Fluoride. Washington, DC: National Academies Press.

20. Rajakumar K, Holick MF, Jeong K et al. (2011) Impact of season and diet on vitamin D status of African American and Caucasian children. Clin Pediatr 50, 493-502.

21. Kumar J, Muntner P, Kaskel F et al. (2009) Prevalence and associations of 25-hydroxyvitamin D deficiency in US children: NHANES 2001-2004. Pediatrics 124, e362-e370.

22. Calvo MS, Whiting SJ \& Barton CN (2004) Vitamin D fortification in the United States and Canada: current status and data needs. Am J Clin Nutr 80, 6 Suppl., 1710S-1716S.

23. Bailey RL, Dodd KW, Goldman JA et al. (2010) Estimation of total usual calcium and vitamin D intakes in the United States. J Nutr 140, 817-822.

24. Rockell J, Skeaff C, Williams S et al. (2006) Serum 25-hydroxyvitamin D concentrations of New Zealanders aged 15 years and older. Osteoporos Int 17, 1382-1389.

25. Rockell JE, Green TJ, Skeaff CM et al. (2005) Season and ethnicity are determinants of serum 25-hydroxyvitamin D concentrations in New Zealand children aged 5-14 y. J Nutr 135, 2602-2608. 
26. Nesby-O'Dell S, Scanlon KS, Cogswell ME et al. (2002) Hypovitaminosis D prevalence and determinants among African American and white women of reproductive age: third National Health and Nutrition Examination Survey, 1988-1994. Am J Clin Nutr 76, 187-192.

27. Matsuoka LY, Wortsman J, Haddad JG et al. (1991) Racial pigmentation and the cutaneous synthesis of vitamin D. Arch Dermatol 127, 536-538.

28. Ogden CL, Carroll MD, Kit BK et al. (2012) Prevalence of obesity and trends in body mass index among US children and adolescents, 1999-2010. JAMA 307, 483-490.
29. Wortsman J, Matsuoka LY, Chen TC et al. (2000) Decreased bioavailability of vitamin D in obesity. Am J Clin Nutr 72, 690-693.

30. Harkness LS \& Cromer BA (2005) Vitamin D deficiency in adolescent females. J Adolesc Health 37, 75.

31. Baranowski T \& Domel S (1994) A cognitive model of children's reporting of food intake. Am J Clin Nutr 59, 1 Suppl., 212S-217S.

32. Rockett H \& Colditz G (1997) Assessing diets of children and adolescents. Am J Clin Nutr 65, 4 Suppl., 1116S-1122S. 